\title{
Potency of integrating three-dimensional cardiac magnetic resonance imaging into electroanatomic mapping to perform catheter ablation in pediatrics
}

\author{
Satoshi Kunimoto ${ }^{1 *}$, Kensuke Yamamoto ${ }^{1}$, Rie Ichikawa ${ }^{2}$, Junji Fukuhara², Masaharu Matsumura ${ }^{2}$, \\ Naokata Sumitomo ${ }^{2}$, Yasuo Okumura', Ichiro Watanabe ${ }^{1}$, Atsushi Hirayama'
}

From 16th Annual SCMR Scientific Sessions

San Francisco, CA, USA. 31 January - 3 February 2013

\section{Background}

Radio-frequency catheter ablation (RFCA) in children is difficult because the size of heart chamber change with age and the catheter made for adults is not appropriate for a child. Three-dimensional (3D) images for electroanatomic mapping (EAM) have been used to create from images of multi-detector computed tomography (MDCT) which need to perform entailed by contrast medium injection and radiation exposure. This study sought to evaluate the usability of 3D Cardiac Magnetic Resonance Image (CMR) in pediatrics cases.

\section{Methods}

Between June 2009 and May 2012, 164 young patients $(10.8 \pm 5.6$ y/o, 89 males) underwent RFCA. 84 patients underwent CMR prior to RFCA without beta blocker and nitroglycerin. CMR was performed by using $1.5-\mathrm{T}$ magnet MR systems (Intera Achieva, Philips Medical Systems) with 5-element cardiac coils. EAM was created by the Carto XP system. CMR results were visually inspected by at least two experienced angiographers whether the image quality of $3 \mathrm{D}$ CMR is sufficient for EAM. We also make a comparison of radiation exposure time between with and without 3D CMR images.

\section{Results}

In spite of dull images because of sinus tachycardia, all CMR images were sufficient for EAM. Radiation exposure time with EAM from CMR was significant shorter than without CMR $(30.0 \pm 2.0$ minutes versus $40.6 \pm 4.1$ minutes, $\mathrm{p}<0.05)$.

\section{Conclusions}

CMR image integration into EAM system was successfully performed in pediatrics patients undergoing RFCA. Procedure of CMR didn't require any medication, contrast medium or radiation exposure. In pediatrics cases, almost all cases are considered an indication for CMR imaging for EAM.

\section{Funding}

None.

\section{Author details}

${ }^{1}$ Division of Cardiology, Nihon University School of Medicine, Tokyo, Japan. 2Division of Pediatrics, Nihon University School of Medicine, Tokyo, Japan.

Published: 30 January 2013

doi:10.1186/1532-429X-15-S1-M9

Cite this article as: Kunimoto et al.: Potency of integrating threedimensional cardiac magnetic resonance imaging into electroanatomic mapping to perform catheter ablation in pediatrics. Journal of Cardiovascular Magnetic Resonance 2013 15(Suppl 1):M9. 